\title{
Peripheral Nerve Grafts and Chondroitinase ABC Application Improves Functional Recovery After Complete Spinal Cord Transection
}

\author{
Amgad Hanna ${ }^{\mathrm{a}, \mathrm{d}}$, Katie E. Kaeppler ${ }^{\mathrm{a}}$, Mark E. Ehlers ${ }^{\mathrm{a}}$, Mahrokh Dadsetan ${ }^{\mathrm{b}}$, Michael J. Yaszemski ${ }^{\mathrm{b}}$, \\ Ross D. Toigo a Junhwa Kima , Euhaa Hwang ${ }^{a}$, Ernesto Bogarin-Miranda ${ }^{a}$, Morgan M. Buchholz ${ }^{\mathrm{a}}$, \\ Alexandra R. Springer ${ }^{\mathrm{a}}$, Daniel J. Hellenbrand ${ }^{\mathrm{a}, \mathrm{c}}$
}

\begin{abstract}
Background: Peripheral nerve grafts (PNGs) in the spinal cord support axonal regeneration and functional recovery. Chondroitinase $\mathrm{ABC}(\mathrm{ChABC})$ has been used to break down chondroitin sulfate proteoglycans (CSPGs) that inhibit axonal regeneration. If ChABC is effectively delivered to the injury site, CSPGs can be broken down so axons can pass through the distal interface between the graft and the spinal cord before CSPG accumulation has an adverse impact on recovery.
\end{abstract}

Methods: In this study, we tested the synergistic effect of using predegenerated PNGs as scaffolds for axonal growth, while delivering $\mathrm{ChABC}$ via oligo-(polyethylene glycol) fumarate (OPF) tubes, to promote functional recovery after complete spinal cord injury in rats.

Results: Our results show that: 1) PNGs anatomically incorporated into the spinal cord after a complete transection, 2) thousands of axons regenerated into the PNGs, 3) cholera toxin-B labeled axons crossed through the graft, and 4) the combination of PNGs and $\mathrm{ChABC}$ restored significantly better locomotor function than controls.

Conclusions: Our study showed that PNGs can establish a good anatomical bridge after SCI and set the stage for functional recovery.

Manuscript accepted for publication July 8, 2013

${ }^{a}$ Department of Neurological Surgery, University of Wisconsin, Madison, WI 53792, USA

${ }^{b}$ Departments of Orthopedic Surgery and Biomedical Engineering, Mayo Clinic, College of Medicine, 200 First Street SW, Rochester MN 55905, USA

${ }^{\mathrm{C}}$ Department of Biomedical Engineering, University of Wisconsin, Madison, WI 53706, USA

${ }^{\mathrm{d}}$ Corresponding author: Amgad Hanna, Department of Neurological Surgery, University of Wisconsin, 600 Highland Avenue, MC 8660, Rm K4/830 CSC, Madison, WI 53792, USA.

Email: a.hanna@neurosurgery.wisc.edu

doi: http://dx.doi.org/10.4021/jnr209w
Keywords: Chondroitinase ABC; Glial scar; Wallerian Degeneration; Oligo-(polyethylene glycol) fumarate

\section{Introduction}

Spinal cord injury (SCI) is a life changing and devastating trauma that impairs many otherwise very active humans across the world. Although there has been significant progress in spinal stabilization and medical care of patients, only minimal progress has been made in spinal cord regeneration or restoration of function.

Impairment due to spinal cord damage is the result of the inability of new axons to grow through the damaged section. This is partially due to an accumulation of upregulated growth inhibitors such as chondroitin sulfate proteoglycans (CSPGs), which form a glial scar [1,2]. The glial scar is a natural response to traumatic injury as it prevents infection, inhibits more widespread damage, and seals the blood brain barrier [3]. However, the densely packed network of tissue and chondroitin sulfate glycosaminoglycan (CS-GAG) chains that make up the glial scar also inhibits axonal regeneration through the injury site [4]. Previously, several studies have shown that injection of chondroitinase $\mathrm{ABC}(\mathrm{ChABC})$, a bacterial enzyme that digests the CS-GAGs, promotes axonal sprouting and functional recovery [5-8]. Most current methods to apply $\mathrm{ChABC}$ involve multiple intrathecal injections, which leads to both problems with diffusion and fluctuation of concentrations of ChABC.

Another problem is tissue degeneration after SCI, leading to cavitation and cyst formation and substantial alteration of the tissue surrounding the lesion. This creates an anatomical gap that axons are unable to cross. There have been several approaches used to bridge this anatomical gap, including poly(lactide-co-glycolide) (PLGA) scaffolds [9, 10], Schwann cells [11], poly(ethylene glycol) hydrogels $[12,13]$, agarose scaffolds $[14,15]$, and peripheral nerve grafts (PNGs) [16-22]. PNGs are a living tissue containing several growth factors that have been shown to promote axonal growth, and cytokines that modulate inflammation $[23,24]$. Before the PNGs are used as scaffolds for axonal 


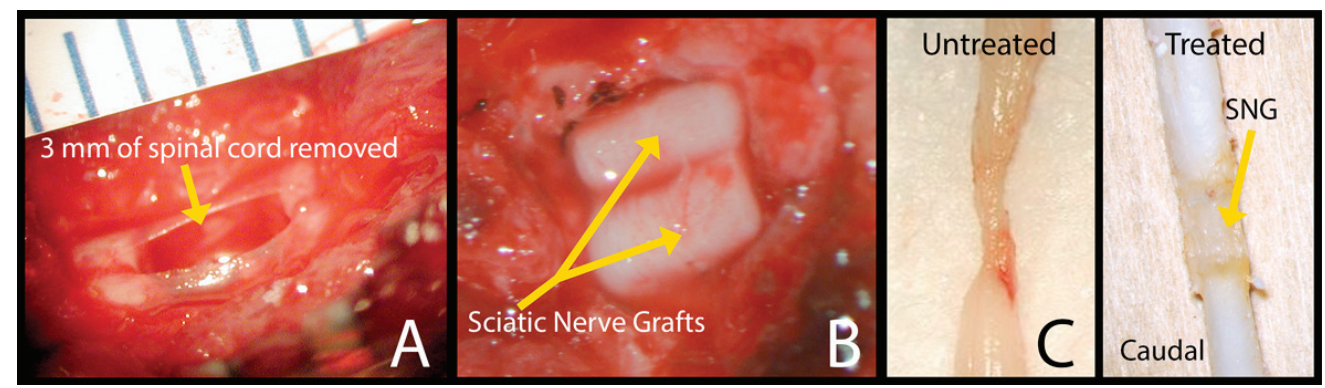

Figure 1. Surgery photos showing (A) $3 \mathrm{~mm}$ of spinal cord removed at the T10 level and (B) 2 pre-degenerated PNGs placed in the spinal canal. (C) Pictures of harvested spinal cords, left side: control and right side: a rat that was treated with an OPF tube releasing $\mathrm{ChABC}$, at 8 weeks.

growth in the spinal cord, they are cut proximally to allow for Wallerian degeneration. During Wallerian degeneration, there is segmentation of damaged axons and myelin, then removal of debris by Schwann cells and immune cells. These two cell types also upregulate several growth factors, which have been shown to enhance axonal regeneration [24]. In an incomplete injury model, several studies have shown that PNGs anatomically incorporate into the spinal cord, induce axonal growth, and promote functional recovery. However, in an incomplete injury model it can be difficult to determine whether the functional improvement is due to regrowth of axons through the graft or plasticity within the intact spinal cord.

Previous researchers have shown that drugs can be released from oligo-(polyethylene glycol) fumarate (OPF) hydrogel for a sustained time period $[25,26]$. The release profile can be tailored by changing the molecular weight of the polyethylene glycol, which changes the amount of crosslinking in the hydrogel [27].

In the present study, we tested the synergistic effect of using predegenerated PNGs as scaffolds for axonal growth after complete thoracic spinal cord transection at T10 in rats, while delivering $\mathrm{ChABC}$ via OPF tubes, to promote functional recovery.

\section{Methods}

\section{Surgical procedures and rat care}

All procedures were performed in accordance with the protocols approved by The University of Wisconsin - Madison Animal Use and Care Committee and followed National Institutes of Health guidelines for the use and care of laboratory animals. Female Sprague Dawley rats weighing 200 - 250 g. were used. For all surgical procedures, rats were anesthetized with $4 \%$ isofluorane, and then maintained on $2-3 \%$ isofluorane for the duration of the surgery. To prevent graft rejection, rats were given $10 \mathrm{mg} / \mathrm{kg} / 24 \mathrm{hr}$ of cyclosporine A via subcutaneous injection for 2 days pre-operatively and for 14 days afterwards, then $100 \mu \mathrm{g} / \mathrm{mL}$ of CsA was administered through drinking water for the remainder of the study $[16,18,28]$.

\section{Specific groups tested}

Rats were divided into four groups. Group 1, used as control, underwent complete spinal cord transection at T10. Group 2, after transection, received two $3 \mathrm{~mm}$ segments of PNGs. In Group 3, the PNGs were wrapped in OPF tubes containing $\mathrm{ChABC}$ in microspheres, before placement in the transection cavity. Group 4 is similar to Group 3 but ChABC was incorporated directly into the hydrogel tubes without microspheres. For the remainder of the paper rats will be referred to in terms of their treatment groups.

\section{PLGA microsphere fabrication}

Microspheres were fabricated using a water-in-oil double emulsion technique. Briefly, $200 \mathrm{mg}$ 50:50 PLGA (Lakeshore Biomaterials) was dissolved in $1 \mathrm{~mL}$ methylene chloride (Sigma Aldrich) in a glass test tube for $2-3 \mathrm{~h}$. While vortexing, $40 \mathrm{U}$ ChABC (Seikagan, bioBusiness) dissolved in $200 \mu \mathrm{L} 0.1 \%$ bovine serum albumin and $2 \mathrm{~mL}$ of $2 \%$ polyvinyl alcohol (PVA) (Sigma Aldrich) was added to the PLGA solution. This PLGA/PVA solution was then poured into a beaker containing $100 \mathrm{~mL} \mathrm{0.3 \%} \mathrm{PVA.} \mathrm{About} 100 \mathrm{~mL} \%$ isopropyl alcohol (Sigma Aldrich) was added to this mix, which was then stirred for at least $1 \mathrm{~h}$ to evaporate the methylene chloride. Microspheres were washed with distilled $\mathrm{H}_{2} \mathrm{O}$, frozen at $-20{ }^{\circ} \mathrm{C}$, and dried under reduced pressure. Microspheres were stored at $-80^{\circ} \mathrm{C}$ until use.

\section{OPF tube fabrication}

Hydrogel tubes were made by dissolving OPF and initiator in distilled $\mathrm{H}_{2} \mathrm{O}$ at $37{ }^{\circ} \mathrm{C}$ for $10 \mathrm{~min}$ according to a previously described method [29]. After centrifugation, N-vinyl pyrrolidinone (NVP) crosslinker was added and vortexed. Fifty milligrams of microspheres containing $\mathrm{ChABC}$ were 
added to $500 \mu \mathrm{L}$ of OPF solution, which was then injected into a glass mold with a metal core and crosslinked using UV light. To fabricate OPF tubing with direct incorporation of $\mathrm{ChABC}, 10 \mathrm{U}$ of $\mathrm{ChABC}$ in $50 \mu \mathrm{L} 0.1 \%$ bovine serum albumin was added to $500 \mu \mathrm{L}$ OPF solution and crosslinked under UV light.

\section{Sciatic nerve surgery}

Rats that were used as sciatic nerve donors had both sciatic nerves cut proximally in the gluteal region. Nerves were allowed to degenerate in vivo for seven days. Right before implantation, the sciatic nerve was harvested by cutting the nerve distally.

\section{Spinal cord injury surgery}

A laminectomy was performed at T10, exposing the spinal cord. The dura was opened, and a $3 \mathrm{~mm}$ section of spinal cord was removed using microscissors and suction (Fig. 1A). For Group 2, two $3 \mathrm{~mm}$ pieces of degenerated sciatic nerve from a donor rat were placed into the transection site to fill the gap and span the circumference of the spinal cord (Fig. 1B). For Groups 3 and 4, a $3 \mathrm{~mm}$ piece of tube was wrapped around the PNGs before implantation. After 8 weeks, the rats were either perfused using 4\% paraformaldehyde or given tracer injections of $1 \%$ conjugated CTB in the sciatic nerve.

\section{Functional recovery assessment}

Functional recovery of hind limb motor activity was assessed using the Basso-Beattie-Bresnahan (BBB) rating scale [30]. Rats were tested before surgery to ensure they are normal (BBB 21) and then tested every seven days post-surgery for 8 weeks. The BBB had to drop to $0-1$ post-injury for the rat to stay in the study. Since the BBB scoring method does not account for frequency of movements, we did a separate analysis based solely on the frequency of hindlimb joint movements. This is a novel technique for assessing functional recovery. All scoring was performed by individuals blinded to the treatment groups.

\section{CTB injection surgery}

After 8 weeks, the sciatic nerves were exposed and $5 \mu \mathrm{L}$ of 1\% CTB conjugated to AlexaFluor 594 (Invitrogen) were injected into the sciatic nerves, and left for a week to allow for proximal transfer through the axons, to assess for regeneration across the PNGs. After one week, rats were perfused with $4 \%$ paraformaldehyde.

\section{Immunohistochemistry}

After perfusion, all spinal cords were placed in $30 \%$ sucrose solution for cryopreservation. When cryopreservation was complete, a section containing the injury site plus $2 \mathrm{~mm}$ rostral and $4 \mathrm{~mm}$ caudal to the injury was removed from the spinal cords. These spinal cord pieces were then frozen and sectioned horizontally $(30 \mu \mathrm{m})$ with a Leica SM2000R microtome. Slides underwent different immunohistochemical procedures for detection of either CTB or neurofilaments (NF). For slides from rats that received CTB injections, sections were rinsed with PBS and cover-slipped (Vector Laboratories, Burlingame, $\mathrm{CA}$ ). For slides from rats that did not receive $\mathrm{CTB}$, sections were treated with mouse anti-neurofilament antibody (1:200, Millipore) and donkey anti-mouse AlexaFluor 350 (1:500, Invitrogen) for detection of neurofilaments. To check for CSPGs, horizontal sections were stained with mouse anti-CS-56 (1:100, Sigma) and to check to broken down CSPSs, sections were stained with mouse anti-1B5 (1:100, MD Bioproducts). Finished slides were examined using a Keyence BZ-9000 microscope. CSPGs were quantified in terms of the percent area stained from $2 \mathrm{~mm}$ rostral to the injury site and $2 \mathrm{~mm}$ caudal to the injury site using Image J software.

\section{Axon counting}

Myelin sheath staining on paraffin-embedded sections was performed with osmium tetroxide to reveal heavily myelinated axons as previously described [31]. The $3 \mathrm{~mm}$ injury site was removed, fixed with osmium tetroxide, then paraffin embedded and $2 \mu \mathrm{m}$ thick transverse sections were taken from the center of the PNG. Sections were placed onto slides and cover-slipped, then pictures of sections were taken using a Keyence BZ-9000 microscope. Myelinated axons were counted using ImageJ software by individuals blinded to the treatment groups.

\section{Statistical analysis}

All quantitative data are presented as means \pm standard error of the mean (SEM). To compare between two specific groups, student's t-test was used. To compare between multiple groups, a one-way analysis of variance (ANOVA) was used to determine statistical differences between results (PRISM). Then, Tukey's post hoc analysis was used to test between specific groups (PRISM). A P value less than 0.05 was considered significant.

\section{Results}

\section{Graft survival and anatomical incorporation into spinal cord}

Eight weeks post-operatively, the spinal cords were harvested and viewed under the operating microscope. Figure $1 \mathrm{C}$ shows 

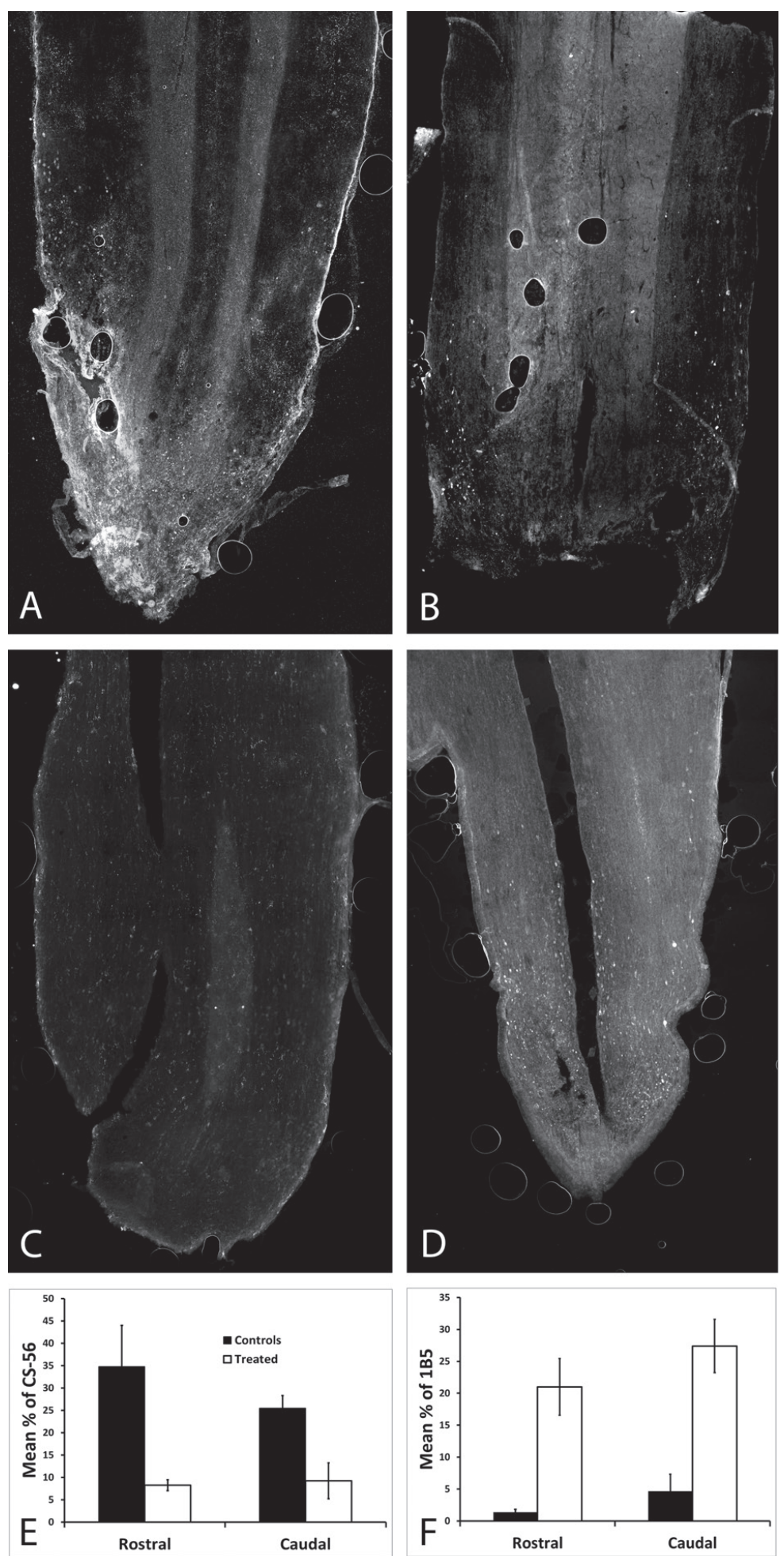

Figure 2. In order to check that ChABC was being released in vivo, full length and broken down CSPGs were labeled on horizontal spinal cord sections harvested 4 weeks post-operatively. (A) CSPGs were abundant in an untreated spinal cord, as opposed to $(B)$ almost none being viewed in the treated spinal cord; both were stained with CS-56. (C) There were no broken down CSPSs in the untreated spinal cord, as opposed to (D) the treated spinal cord which had a significant amount of broken down CSPGs $(p<0.05)$, both were stained with 1B5. (E) The percent area stained for CS-56 was significantly higher both rostral and caudal in the untreated spinal cord $(p<0.05)$. ( $F)$ The percent area stained for 1 B5 was significantly higher both rostral and caudal in the ChABC treated spinal cord. 


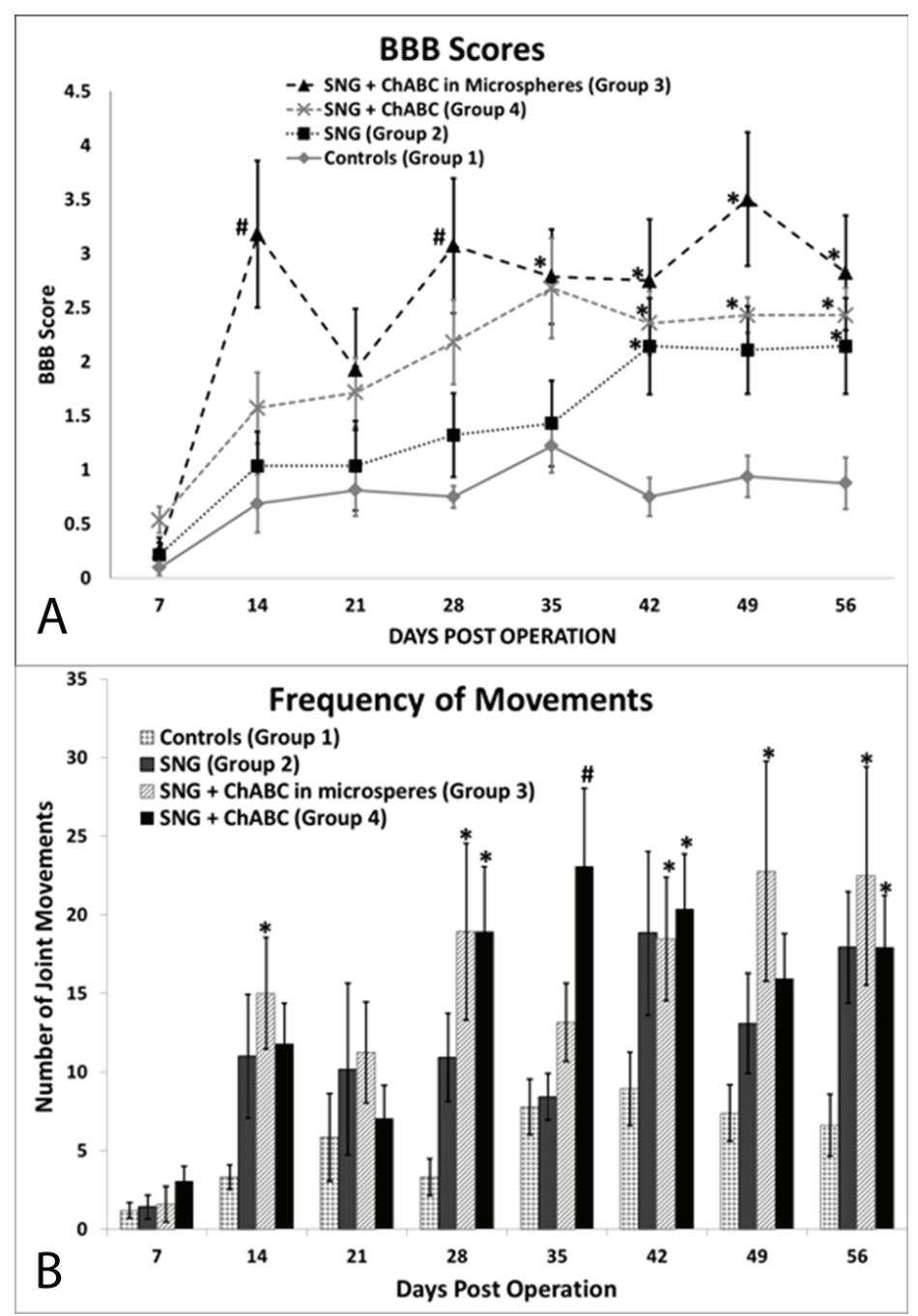

Figure 3. Functional recovery assessment results. (A) Hindlimb locomotor BBB scores show that all groups treated with PNGs did significantly better than controls, (B) ChABC treated groups moved their hindlimbs significantly more than controls on day 56. *Denotes $\mathrm{P}<0.05$ compared to control, \# denotes $\mathrm{P}<0.05$ compared to control and to PNG only group, error bars represent \pm SEM.

two PFA fixed spinal cords with no sign of infection or graft rejection. In most rats, the PNGs were well apposed both distally and proximally (Fig. 1C). There were 15 control rats (Group 1) of which two appeared to be unhealthy with a large area of necrotic tissue rostral to the injury site when harvested. These two rats were removed from all analysis data. Each of the three treated groups had one rat that did not have appropriate anatomical incorporation of the PNG into the spinal cord. These three rats were also removed from all data analysis. The final numbers of rats used for each group are: Group $1 \mathrm{~N}=13$, Group $2 \mathrm{~N}=12$, Group $3 \mathrm{~N}=13$, and Group $4 \mathrm{~N}=13$.

\section{ChABC activity in vivo}

Rats received a complete $\mathrm{SCI}$ and were either given no treatment or given an OPF tube and microspheres releasing $\mathrm{ChABC}$ and harvested 4 weeks post-operatively. In order to test for ChABC activity in vivo, full length and broken down CS-GAGs were labeled on $30 \mu \mathrm{m}$ horizontal sections. The percent area, both rostral and caudal, stained with CS-56 for full length CSPGs was significantly less in the rats treated with ChABC (Fig. 2A, B, E). The percent area, both rostral and caudal, stained with 1B5 for broken down CSPGs was significantly higher in the rats treated with ChABC (Fig. 2C, D, F).

Functional recovery assessment after complete spinal cord transection (Fig. 3)

Before spinal cord surgery, all rats had a BBB score of 21 (data not shown). After complete spinal cord transection, all rats dropped to a BBB score of one or less (Fig. 3A). The rats treated with PNGs (Group 2) scored significantly higher than the 


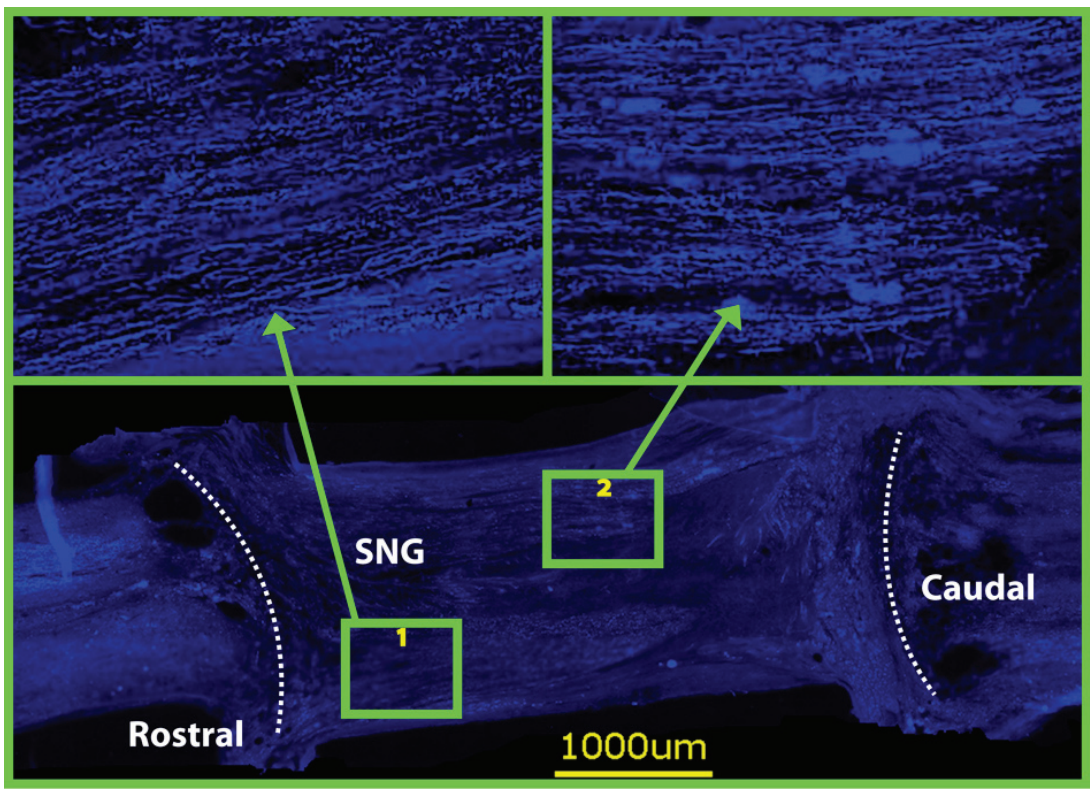

Figure 4. Neurofilament staining on horizontal sections at 8 weeks revealed that both grafts were densely populated with axons. Dotted lines indicate graft spinal cord interface.

controls on days 42,49 , and 56 . ChABC treated rats did significantly better than the control group; Group 3 was significantly higher than Group 1 on all days except days 7 and 21 and Group 4 was significantly higher than Group 1 on all days except day 7. On all days after day 7, the ChABC groups did better than the rats that only received the PNGs (Group 2). Group 3 was significantly higher than Group 2 on days 14, 18, 35 and Group 4 was significantly higher than Group 2 on day 35 . Although there is a trend showing that Group 3 did better than Group 4, there was no statistical significance.

Using our new frequency scoring, we scored the total number of hindlimb movements separately (Fig. 3B). After SCI, all groups dropped to less than five hindlimb movements on day 7. All treated groups had more movements than the controls at all time points, and all three treated groups were significantly higher than Group 1, on day 56 . Group 3 had significantly more movements than Group 1 on all days except days 7, 21 and 35 . Group 4 had significantly more movements than Group 1 on all days except days 7 and 21 . There was no statistical significance between the three treated groups.

\section{Axonal regeneration into peripheral nerve grafts}

Numerous regenerated axons were observed with NF stain at 8 weeks (Fig. 4). This was a consistent finding in all the NFstained grafts from all treated groups. Osmium tetroxide was used to stain the myelin (Fig. 5A, B, C). The myelinated axons in the PNGs were counted in 4 rats from each group (Fig. 5D). The mean number of axons in each group were; Group 2 $(2,640)$, Group $3(2,010)$ and Group $4(2,740)$. There was no significant difference between these groups.
Regenerated axons extend through the peripheral nerve graft

The CTB was successfully taken up by the sciatic nerve in 22 rats and seen caudal to the graft. In the control rats, the CTB was never seen rostral to the lesion (Fig. 6A, Fig. 7). CTB was observed in the PNGs and in the spinal cord rostral to it in 5 out of 6 rats from Group 2, 4 out of 6 rats from Group 3, and 4 out of 5 rats from Group 4 (Fig. 6B-D, Fig. 7). It was difficult, however, to count individually labeled axons because the CTB was granular in appearance.

\section{Discussion}

There have been several promising results when using PNGs in incomplete spinal cord injuries [16-18]. However, when using an incomplete injury model, it can be difficult to inflict the exact same injury, so that all rats start the recovery process with the same level of function. Another challenging aspect when using an incomplete model is determining whether the functional recovery was actually restored via axonal regeneration through the PNG or due to plasticity within the intact spinal cord. In this study, we used a complete injury requiring all rats to start recovery at the same level of function and all functional recovery to occur via reconnection through the graft. Some rats were excluded from the analysis because at the time of harvesting the spinal cord did not look healthy or the graft did not anatomically incorporate into the spinal cord. We are not sure if this was due to infection, graft rejection or technical procedures. Currently, we are testing 


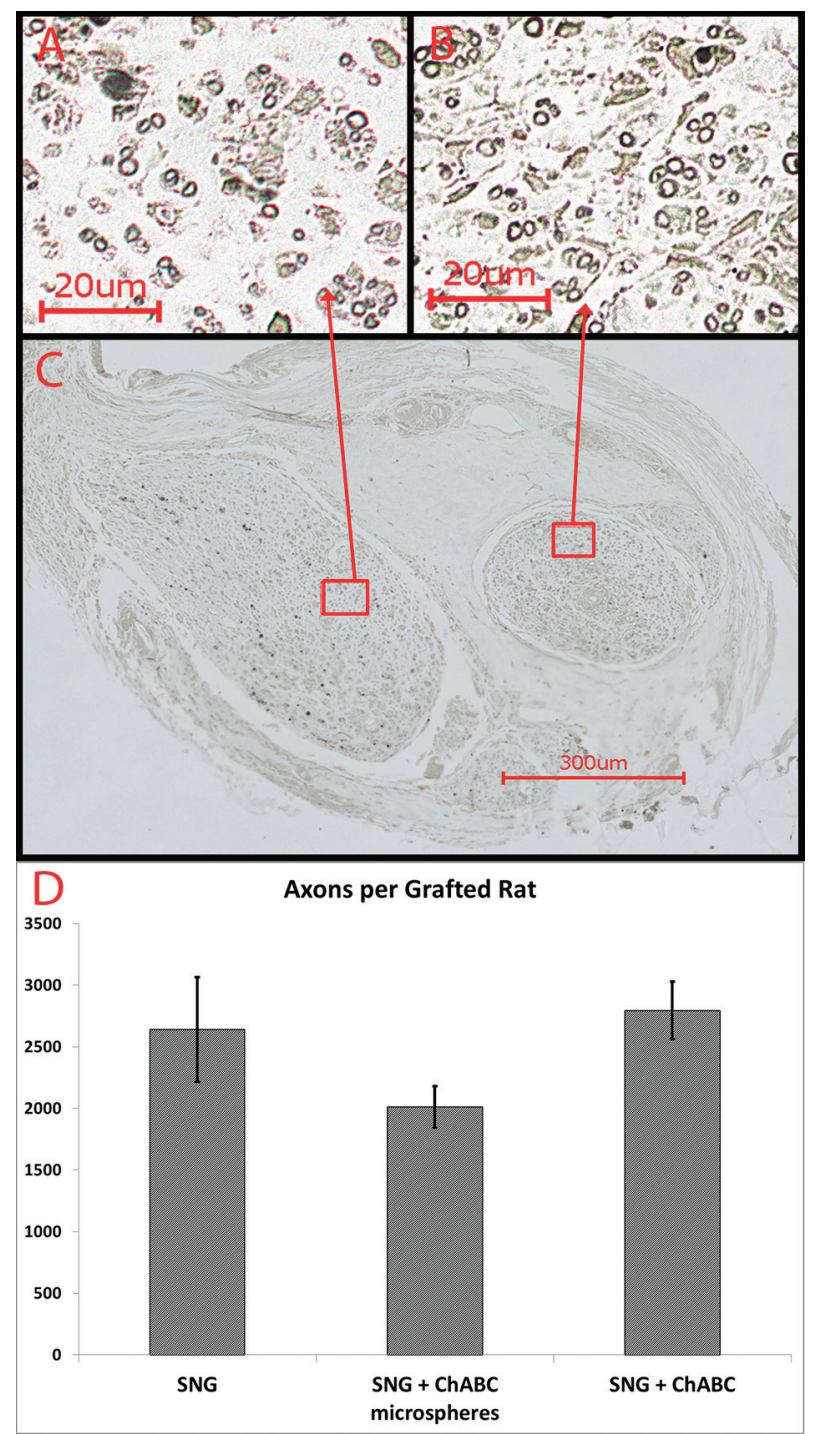

Figure 5. (A-B) Myelin staining with osmium tetroxide revealed numerous heavily myelinated axons of small and large caliber that regenerated into both PNGs, shown on a transverse section (C) through the midpoint of the PNGs at eight weeks. (D) Number of heavily mylinated axons in the PNGs ( $n=4$ from each group). Error Bars Represent \pm SEM.

Lewis inbred rats (Harlan) to assess their ability to resist graft rejection.

\section{Induced functional recovery after complete spinal cord injury}

We show that ChABC application to a complete SCI promotes regrowth of acutely injured axons through the distal interface aspect of the PNG into host spinal cord, contributing to improved functional recovery. These results are consistent with previous studies, which have shown axons growing through the distal interface of the PNG [16-18].

One of the most important measures of the efficacy of a particular regeneration treatment, especially in nervous sys- tem injuries, is behavioral testing. The results of our BBB scoring clearly show functional improvement when a PNG is used to bridge the injury site, and the application of ChABC was shown to improve these scores even further. Furthermore, although experimental averages were around 3.5, it is noteworthy that some individual subjects scored as high as 7 with some signs of weight bearing on their knees.

Throughout the span of our study, we came to find that BBB scores alone were not enough to completely reflect the different levels of functional recovery seen between the various groups. We developed a new system to record each individual joint movement over a four minute period. The decision to include this frequency data resulted from the observation that, many rats were repeatedly moving the same joint 


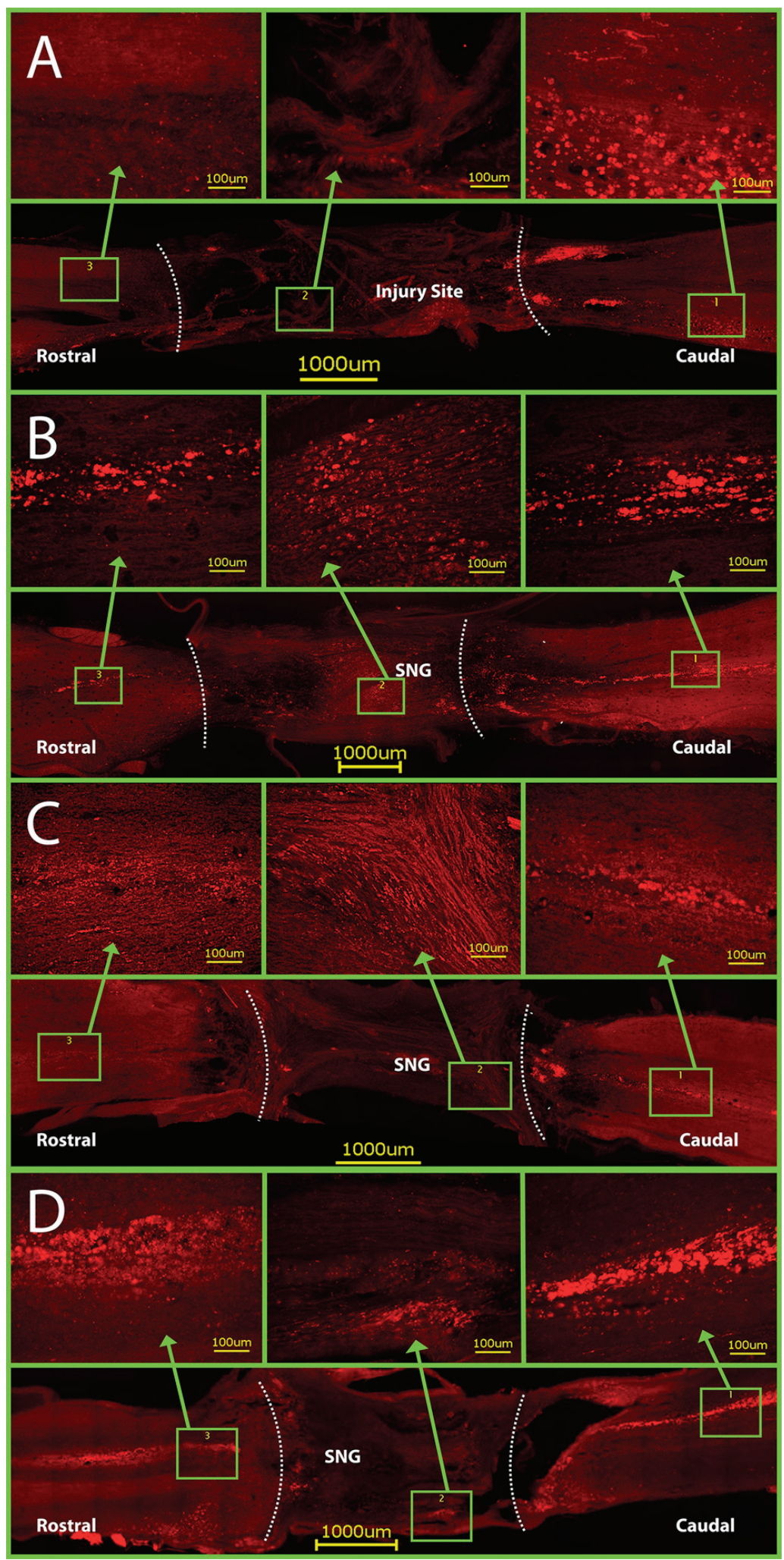

Figure 6. CTB staining in horizontal sections of spinal cords. Dotted lines indicate graft- spinal cord interface. (A) Control rats have CTB staining only caudal to the injury site. Conversely, CTB crossed through the PNGs and into the rostral spinal cord in groups with: (B) only a PNG, (C) a PNG with OPF tubes releasing ChABC from microspheres, and (D) a PNG with just an OPF tube releasing ChABC. Note: the graft- spinal cord interface on the caudal side of Figure $D$ was torn slightly during spinal cord harvest. 


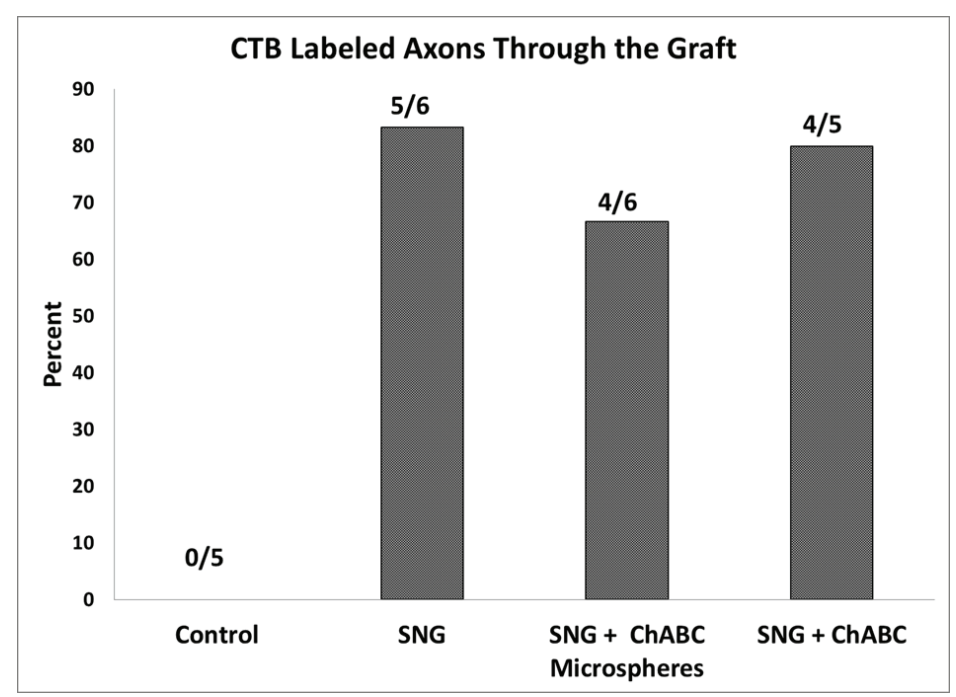

Figure 7. Quantification of rats that have positive CTB labeled axons on the rostral side of the PNGs. All 22 rats listed had positive CTB staining caudal to the PNGs.

to the same extent. The early stages of the BBB assessment scale only take into account types of movement (slight or extensive) and number of joints moved, but not the frequency of movements. By including frequency calculations, the data confirmed the differences between the groups (Fig. 3B).

\section{Axonal regeneration through a completely transected spinal cord}

Our data from the myelin staining shows that there was no significant difference in axon counts between groups 2,3 and 4. Group 1 data was not shown because the injury site consisted only of scar tissue, with no observed axons. The aim of chondroitinase application was not to get axons to grow into the PNGs but rather to help them exit the graft into the spinal cord, which may explain why there was no significant difference in the number of myelinated axons between the groups.

Furthermore, the use of CTB showed that axons regrew through the injury site in the majority of the rats with PNGs, while the CTB never crossed into the injury of control rats. As the CTB was conjugated, no nonspecific staining can occur and the only seen CTB would be that which travelled with the axon, through the entire injury site. The morphology of the CTB staining rostral to the graft appears slightly different than the labeled axons on the caudal side. Previously, Alto et al. had similar results distal to an injury site and they showed with double labeling for myelin-associated glycoprotein and $\mathrm{CTB}$ that axons regenerating into and beyond the lesion site remained demyelinated or sparsely myelinated [32]. Not having proper myelination may explain the difference we see in CTB labeled axons. Also, improper myelination may be limiting the amount of functional recovery we are able to restore. In future projects, we will check for myelination of labeled axons distal to the graft.

\section{OPF tubes for ChABC delivery}

It is well documented that regeneration of injured axons after an acute or chronic SCI can be facilitated by the application of $\mathrm{ChABC}[8,18]$. In this study, we did have more functional recovery in the ChABC treated rats, although it was not always significant. We also did not find a significant difference in improvement whether $\mathrm{ChABC}$ was directly released from the tubes or through microspheres (groups 4 and 3 respectively). To our knowledge this is the first report of delivering ChABC through OPF hydrogel tubes. Hydrogel tubes carry the advantage of being familiar to surgeons and being technically easy to implant. The same is true for PNGs. More detailed in vitro studies may more accurately reflect ChABC release from the OPF tubes, however this may not exactly reflect how it would react in vivo.

\section{Conclusions}

Our study showed that PNGs can establish a good anatomical bridge after SCI and set the stage for functional recovery. The complete injury model can be used to test the efficacy of therapeutic proteins such as ChABC. OPF tubes are a good vehicle for ChABC delivery and are easy to manipulate surgically, which makes future clinical application very feasible. Future studies should tailor the release profile of ChABC from OPF hydrogel and test the bioactivity of the released ChABC.

\section{Acknowledgement}

We wish to acknowledge Joseph A. Hardin in Experimental Pathology with the University of Wisconsin. Carbone Can- 
cer Center, N.C.I. \#P30CA014520, for help with Immunochemistry.

\section{References}

1. McKeon RJ, Schreiber RC, Rudge JS, Silver J. Reduction of neurite outgrowth in a model of glial scarring following CNS injury is correlated with the expression of inhibitory molecules on reactive astrocytes. J Neurosci. 1991;11(11):3398-3411.

2. Fawcett JW, Asher RA. The glial scar and central nervous system repair. Brain Res Bull. 1999;49(6):377-391.

3. Shechter R, London A, Varol C, Raposo C, Cusimano M, Yovel G, Rolls A, et al. Infiltrating blood-derived macrophages are vital cells playing an anti-inflammatory role in recovery from spinal cord injury in mice. PLoS Med. 2009;6(7):e1000113.

4. Siebert JR, Osterhout DJ. The inhibitory effects of chondroitin sulfate proteoglycans on oligodendrocytes. J Neurochem. 2011;119(1):176-188.

5. Bradbury EJ, Moon LD, Popat RJ, King VR, Bennett GS, Patel PN, Fawcett JW, et al. Chondroitinase ABC promotes functional recovery after spinal cord injury. Nature. 2002;416(6881):636-640.

6. Barritt AW, Davies M, Marchand F, Hartley R, Grist J, Yip P, McMahon SB, et al. Chondroitinase ABC promotes sprouting of intact and injured spinal systems after spinal cord injury. J Neurosci. 2006;26(42):1085610867.

7. Caggiano AO, Zimber MP, Ganguly A, Blight AR, Gruskin EA. Chondroitinase ABCI improves locomotion and bladder function following contusion injury of the rat spinal cord. J Neurotrauma. 2005;22(2):226-239.

8. Lee H, McKeon RJ, Bellamkonda RV. Sustained delivery of thermostabilized chABC enhances axonal sprouting and functional recovery after spinal cord injury. Proc Natl Acad Sci U S A. 2010;107(8):3340-3345.

9. Fan J, Zhang H, He J, Xiao Z, Chen B, Xiaodan J, Dai $\mathrm{J}$, et al. Neural regrowth induced by PLGA nerve conduits and neurotrophin-3 in rats with complete spinal cord transection. J Biomed Mater Res B Appl Biomater. 2011;97(2):271-277.

10. De Laporte L, Yan AL, Shea LD. Local gene delivery from ECM-coated poly(lactide-co-glycolide) multiple channel bridges after spinal cord injury. Biomaterials. 2009;30(12):2361-2368.

11. Xu XM, Zhang SX, Li H, Aebischer P, Bunge MB. Regrowth of axons into the distal spinal cord through a Schwann-cell-seeded mini-channel implanted into hemisected adult rat spinal cord. Eur J Neurosci. 1999;11(5):1723-1740.

12. Namba RM, Cole AA, Bjugstad KB, Mahoney MJ. Development of porous PEG hydrogels that enable effi- cient, uniform cell-seeding and permit early neural process extension. Acta Biomater. 2009;5(6):1884-1897.

13. Krsko P, McCann TE, Thach TT, Laabs TL, Geller HM, Libera MR. Length-scale mediated adhesion and directed growth of neural cells by surface-patterned poly(ethylene glycol) hydrogels. Biomaterials. 2009;30(5):721-729.

14. Gros T, Sakamoto JS, Blesch A, Havton LA, Tuszynski MH. Regeneration of long-tract axons through sites of spinal cord injury using templated agarose scaffolds. Biomaterials. 2010;31(26):6719-6729.

15. Stokols S, Sakamoto J, Breckon C, Holt T, Weiss J, Tuszynski MH. Templated agarose scaffolds support linear axonal regeneration. Tissue Eng. 2006;12(10):27772787.

16. Houle JD, Tom VJ, Mayes D, Wagoner G, Phillips N, Silver J. Combining an autologous peripheral nervous system "bridge" and matrix modification by chondroitinase allows robust, functional regeneration beyond a hemisection lesion of the adult rat spinal cord. J Neurosci. 2006;26(28):7405-7415.

17. Cote MP, Hanna A, Lemay MA, Ollivier-Lanvin K, Santi L, Miller K, Monaghan R, et al. Peripheral nerve grafts after cervical spinal cord injury in adult cats. Exp Neurol. 2010;225(1):173-182.

18. Tom VJ, Sandrow-Feinberg HR, Miller K, Santi L, Connors T, Lemay MA, Houle JD. Combining peripheral nerve grafts and chondroitinase promotes functional axonal regeneration in the chronically injured spinal cord. J Neurosci. 2009;29(47):14881-14890.

19. Tadie M, Liu S, Robert R, Guiheneuc P, Pereon Y, Perrouin-Verbe B, Mathe JF. Partial return of motor function in paralyzed legs after surgical bypass of the lesion site by nerve autografts three years after spinal cord injury. $\mathrm{J}$ Neurotrauma. 2002;19(8):909-916.

20. David S, Aguayo AJ. Axonal elongation into peripheral nervous system "bridges" after central nervous system injury in adult rats. Science. 1981;214(4523):931-933.

21. Tom VJ, Houle JD. Intraspinal microinjection of chondroitinase $\mathrm{ABC}$ following injury promotes axonal regeneration out of a peripheral nerve graft bridge. Exp Neurol. 2008;211(1):315-319.

22. Alilain WJ, Horn KP, Hu H, Dick TE, Silver J. Functional regeneration of respiratory pathways after spinal cord injury. Nature. 2011;475(7355):196-200.

23. Chen Q, Smith GM, Shine HD. Immune activation is required for NT-3-induced axonal plasticity in chronic spinal cord injury. Exp Neurol. 2008;209(2):497-509.

24. Barrette B, Calvo E, Vallieres N, Lacroix S. Transcriptional profiling of the injured sciatic nerve of mice carrying the Wld(S) mutant gene: identification of genes involved in neuroprotection, neuroinflammation, and nerve regeneration. Brain Behav Immun. 2010;24(8):12541267. 
25. Kasper FK, Kushibiki T, Kimura Y, Mikos AG, Tabata Y. In vivo release of plasmid DNA from composites of oligo(poly(ethylene glycol)fumarate) and cationized gelatin microspheres. J Control Release. 2005;107(3):547561.

26. Kasper FK, Jerkins E, Tanahashi K, Barry MA, Tabata Y, Mikos AG. Characterization of DNA release from composites of oligo(poly(ethylene glycol) fumarate) and cationized gelatin microspheres in vitro. J Biomed Mater Res A. 2006;78(4):823-835.

27. Dadsetan M, Szatkowski JP, Yaszemski MJ, Lu L. Characterization of photo-cross-linked oligo[poly(ethylene glycol) fumarate] hydrogels for cartilage tissue engineering. Biomacromolecules. 2007;8(5):1702-1709.

28. Tobias CA, Shumsky JS, Shibata M, Tuszynski MH, Fischer I, Tessler A, Murray M. Delayed grafting of BDNF and NT-3 producing fibroblasts into the injured spinal cord stimulates sprouting, partially rescues axotomized red nucleus neurons from loss and atrophy, and provides limited regeneration. Exp Neurol. 2003;184(1):97-113.

29. Dadsetan M, Knight AM, Lu L, Windebank AJ, Yaszemski MJ. Stimulation of neurite outgrowth using positively charged hydrogels. Biomaterials. 2009;30(2324):3874-3881.

30. Basso DM, Beattie MS, Bresnahan JC. A sensitive and reliable locomotor rating scale for open field testing in rats. J Neurotrauma. 1995;12(1):1-21.

31. Di Scipio F, Raimondo S, Tos P, Geuna S. A simple protocol for paraffin-embedded myelin sheath staining with osmium tetroxide for light microscope observation. Microsc Res Tech. 2008;71(7):497-502.

32. Alto LT, Havton LA, Conner JM, Hollis ER, 2nd, Blesch A, Tuszynski $\mathrm{MH}$. Chemotropic guidance facilitates axonal regeneration and synapse formation after spinal cord injury. Nat Neurosci. 2009;12(9):1106-1113. 